\title{
OKOUNKOV BODIES FOR AMPLE LINE BUNDLES WITH APPLICATIONS TO MULTIPLICITIES FOR GROUP REPRESENTATIONS
}

\author{
HENRIK SEPPÄNEN*
}

\begin{abstract}
Let $\mathscr{L} \rightarrow X$ be an ample line bundle over a complex normal projective variety $X$. We construct a flag $X_{0} \subseteq X_{1} \subseteq \cdots \subseteq X_{n}=$ $X$ of subvarieties for which the associated Okounkov body for $\mathscr{L}$ is a rational polytope. In the case when $X$ is a homogeneous surface, and the pseudoeffective cone of $X$ is rational polyhedral, we also show that the global Okounkov body is a rational polyhedral cone if the flag of subvarieties is suitably chosen. Finally, we provide an application to the asymptotic study of group representations.
\end{abstract}

\section{INTRODUCTION}

The Okounkov body of a line bundle $\mathscr{L} \rightarrow X$ over an $n$-dimensional complex variety is a convex set in $\mathbb{R}^{n}$ which carries information about the section ring $\bigoplus_{k=0}^{\infty} H^{0}\left(X, \mathscr{L}^{k}\right)$ of $\mathscr{L}$. The idea is to construct a valuationlike function (meaning that it has the properties of a valuation in the ringtheoretic sense, even if it only defined on homogeneous elements)

$$
v: \bigsqcup_{k \in \mathbb{N}} H^{0}\left(X, \mathscr{L}^{k}\right) \backslash\{0\} \rightarrow \mathbb{N}_{0}^{n}
$$

and define the semigroup

$$
S(\mathscr{L}, v):=\left\{(k, v(s)) \mid s \in H^{0}\left(X, \mathscr{L}^{k}\right) \backslash\{0\}\right\} .
$$

The associated Okounkov body is then defined as

$$
\Delta(\mathscr{L}, v):=\overline{\operatorname{conv}}\left\{\frac{1}{k}(k, v(s)) \mid s \in H^{0}\left(X, \mathscr{L}^{k}\right) \backslash\{0\}\right\} .
$$

Roughly speaking, the function $v$ will be defined as the successive orders of vanishing of sections along a flag $X_{0} \subseteq X_{1} \subseteq \cdots \subseteq X_{n-1} \subseteq X_{n}=X$ of irreducible nonsingular subvarieties such that $X_{i}$ has dimension $i$.

These bodies were introduced by Okounkov in 096 from a representationtheoretic point of view. However, he only considered the semigroup defined by the values of $U$-invariants, where $U$ is the unipotent radical of a Borel subgroup, $B$, of $G$. It turned out that the elements of height $k$ in this semigroup carry information about the decomposition under $G$ of the $k$ th piece, $H^{0}\left(X, \mathscr{L}^{k}\right)$, and that the Euclidean volumes of certain slices of the associated convex body describe the asymptotics of the decomposition.

Date: June 2, 2021.

Key words and phrases. Ample line bundle, Okounkov body, irreducible representation, branching laws.

*supported by the DFG Priority Programme 1388 "Representation Theory". 
It has since then become an interesting problem per se to study the semigroups and associated convex bodies defined by considering the values of all sections of a line bundle, without the assumption of a group action ([LM09]), and their relation to the geometry of the line bundle $\mathscr{L}$. One crucial connection between the section ring of $\mathscr{L}$ and the Okounkov body $\Delta(\mathscr{L}, v)$ is the identity

$$
\operatorname{vol} \Delta(\mathscr{L}, v)=\lim _{k \rightarrow \infty} \frac{\operatorname{dim} H^{0}\left(X, \mathscr{L}^{k}\right)}{k^{n}},
$$

which holds for the linear series of a big line bundle over an $n$-dimensional complex projective variety $X$ (see [LM09]).

A fundamental question to ask is whether $\Delta(\mathscr{L}, v)$ is a rational polytope, i.e., the convex hull of finitely many points in $\mathbb{Q}^{n}$. This was conjectured by Okounkov in [096 in his setting where he only considered $U$-invariants. It is true in some cases, e.g., equivariant line bundles over toric varieties. This follows from recent results by Kaveh and Khovanskii where they consider a setting that generalizes Okounkov's, namely group representations on graded algebras of meromorphic functions on $X$, cf. [KK10]. In fact, the Okounkov body of a torus-equivariant line bundle over a projective toric variety equals the moment polytope (cf. [B86]) associated to the graded representation. Kaveh ([K11]) has studied this problem for a line bundle over a full flag variety $G / B$. He considers an Okounkov body defined by a valuation determined by a local system of coordinates on a Bott-Samelson resolution, and shows that this Okounkov body is a polytope by identifying it with a Littelmann string polyope.

It is, however, not true in general that $\Delta(L, v)$ is a rational polytope. A counterexample can be found in [LM09, Section 6.3].

In this paper, we prove that if $\mathscr{L}$ is ample, there exists a flag of nonsingular irreducible subvarieties $X_{0} \subseteq X_{1} \subseteq \cdots \subseteq X_{n}:=X$ for which the associated Okounkov body is a rational polytope. In the case when $X$ is a homogenous surface, we can say a little more: If the pseudoeffective cone $\overline{\mathrm{Eff}}(X)$ is a rational polyhedral cone, and $D$ is a fixed ample divisor on $D$, then for a very general choice of flag $X_{0} \subseteq X_{1}$, where $X_{1}$ is an irreducible divisor in the linear system $|D|$, the global Okonkov body of $X$ with respect to this flag is a rational polyhedral cone.

We would like to point out that the first result, Theorem 3.3, which deals with a given ample line bundle, has also been obtained independently - but with a different proof - by Anderson, Küronya and Lozovanu in [AKL12. In fact, they even prove the result under the weaker assumption of having a semi-ample line bundle. We like to present an alternative proof for this known result nevertheless, since the proof can be adapted to the global situation for homogeneous surfaces in Section 4. Moreover, we are working on pushing these techniques a little further in a more general context in a current joint project with D. Schmitz ([SS14]).

In the final section we address the original motivation for Okounkov, namely that of studying multiplicities for group representations, by applying the result to certain GIT-quotients; thus providing a "polyhedral" expression 
for asymptotic branching laws, which is by now a well established goal in representation theory.

Acknowledgement. The author would like to thank Jacopo Gandini and Joachim Hilgert for interesting discussions on Okounkov bodies, as well as Shin-Yao Jow and David Schmitz for helpful comments on an earlier version of this manuscript.

\section{Flags of nORMal SUbVarieties}

Let $X$ be a normal complex variety with singular locus $\operatorname{sing}(X)$ and set of regular points $X^{\text {reg }}$, and let $D \subseteq X$ be a prime Cartier divisor given as the zero set of the section $\xi \in H^{0}\left(X, \mathcal{O}_{X}(D)\right)$. If $Y \subseteq X$ is any effective Cartier divisor of $X$ and $s \in H^{0}\left(X, \mathcal{O}_{X}(Y)\right)$ is a section of $\mathcal{O}_{X}(Y)$ that vanishes to order $a \in \mathbb{N}_{0}$ along $D$, the quotient $s / \xi^{a}$ defines a meromorphic section of $\mathcal{O}_{X}(D-a Y)$. In fact, this is even a regular section. Indeed, if $\left\{U_{\alpha}\right\}$ is a finite open cover of $X$ such that $\mathcal{O}_{X}(D)$ is trivial over each $U_{\alpha}$ and the section $\xi$ is represented by the regular function $f_{\alpha} \in \mathcal{O}_{X}\left(U_{\alpha}\right)$ on $U_{\alpha}$ via this local trivialization, then the section $s / \xi^{a}$ is regular on the set

$$
V_{\alpha}:=\left\{x \in D \cap U_{\alpha}^{\mathrm{reg}} \mid d f_{\alpha}(x) \neq 0\right\},
$$

where $U_{\alpha}^{\text {reg }}:=U_{\alpha} \cap X^{\mathrm{reg}}$, since $f_{\alpha}$ vanishes to order one along $D$ on this set, and any local trivialization of $s$ vanishes to order $a$. Since $s / \xi^{a}$ is clearly regular outside $D$, it follows that it is regular outside the closed set

$$
Z:=\bigcup_{\alpha} D \cap \overline{\left\{x \in U_{\alpha}^{\mathrm{reg}} \mid d f_{\alpha}(x)=0\right\}} \cup(D \cap \operatorname{sing}(X)) .
$$

Since $\xi$ vanishes to order one along $D$, each subset $D \cap \overline{\left\{x \in U_{\alpha}^{\text {reg }} \mid d f_{\alpha}(x)=0\right\}}$ is a proper closed subset of $D$, and hence of codimension at least two in $X$. Moreover, since $X$ is normal, the singular locus $\operatorname{sing}(X)$ is also of codimension at least two. Hence, $Z$ is of codimension at least two in $X$. Since $s / \xi^{a}$ is regular outside $Z$, it thus follows from the normality of $X$ that $s / \xi^{a}$ is regular everywhere.

Flag of normal varieties, construction of Okounkov body. Assume now that we have a flag $X_{0} \subseteq X_{1} \subseteq X_{n-1} \subseteq X_{n}=X$ of irreducible normal subvarieties with $\operatorname{dim} X_{i}=i$ for $i=0, \ldots, n$ and that for each $i=2, \ldots, n$, the subvariety $X_{i-1}$ is a Cartier divisor of $X_{i}$ given as the zero set of a section $\xi_{n-i+1} \in \mathcal{O}\left(X_{i-1}\right.$. Let $\mathscr{L}_{n-i+1}$ be the corresponding line bundle over $X_{i}$. If $\mathscr{L}$ is an effective Cartier divisor of $X$, we define a valuation-like function

$$
v: \bigsqcup_{k=0}^{\infty} H^{0}\left(X, \mathscr{L}^{k}\right) \backslash\{0\} \rightarrow \mathbb{N}_{0}^{n}
$$

inductively as follows.

If $s \in H^{0}\left(X, \mathcal{L}^{k}\right)$ vanishes to order $a_{n}$ along $X_{n-1}$, then, by the above, $s / \xi_{1}^{a_{n}}$ is a regular section of $H^{0}\left(X, \mathscr{L}^{k} \otimes \mathscr{L}_{1}^{a_{n}}\right)$ which does not vanish identically on $X_{n-1}$. Assume now that $a_{n}, \ldots, a_{n-i+1}$ are defined in such a way that $s /\left(\xi_{1}^{a_{1}} \cdots \xi_{i}^{a_{n-i+1}}\right)$ defines a regular section of $\mathscr{L}^{k} \otimes \mathscr{L}_{1}^{-a_{1}} \otimes \cdots \otimes$ $\mathscr{L}_{i}^{-a_{n-i+1}}$ over $X_{n-i+1}$ which does not vanish identically on $X_{n-i}$. Let $a_{n-i}$ be the order of vanishing along $X_{n-i-1}$ of the restriction to $X_{n-i}$ of $s /\left(\xi_{1}^{-a_{1}} \cdots \xi_{i}^{-a_{n-i+1}}\right)$. By the first paragraph of this section, $s /\left(\xi_{1}^{-a_{1}} \cdots \xi_{i+1}^{-a_{n-i}}\right)$ 
is a regular section of $\mathscr{L}^{k} \otimes \mathscr{L}_{1}^{-a_{1}} \otimes \cdots \otimes \mathscr{L}_{i+1}^{-a_{n-i}}$ over $X_{n-i}$ which does vanish identically on $X_{n-i-1}$.

We then define

$$
v(s):=\left(a_{1}, \ldots, a_{n}\right) \in \mathbb{N}_{0}^{n} .
$$

Then $v$ is an $\mathbb{N}_{0}^{n}$-valued valuation-like function on $\bigsqcup_{k=0}^{\infty} H^{0}\left(X, \mathscr{L}^{k}\right) \backslash\{0\}$ with respect to the inverse lexicographic order on $\mathbb{N}_{0}^{n}$. We now use $v$ to define the semigroup

$$
S(\mathscr{L}, v):=\left\{(k, v(s)) \mid s \in H^{0}\left(X, \mathscr{L}^{k} \backslash\{0\}\right)\right\} \subseteq \mathbb{N}_{0} \times \mathbb{N}_{0}^{n}
$$

and the closed convex cone

$$
C(\mathscr{L}, v) \subseteq \mathbb{R} \oplus \mathbb{R}^{n}
$$

generated by the semigroup $S(\mathscr{L}, v)$. Finally, the Okounkov body is

$$
\Delta(\mathscr{L}, v)=\Delta_{X}(\mathscr{L}):=C(\mathscr{L}, v) \cap\left(\{1\} \times \mathbb{R}^{n}\right) .
$$

Remark 2.1. The construction can also be carried out by using a local trivialization of $\mathscr{L}$ to embed the section ring $R(L):=\bigoplus_{k=0}^{\infty} H^{0}\left(X, \mathscr{L}^{k}\right)$ into the ring of rational functions $\mathbb{C}(X)$ and to use the flag to define a proper $\mathbb{Z}^{n}$-valued valuation on the ring $\mathbb{C}(X)$. For this, the assumption that $X_{i-1}$ be a Cartier divisor of $X_{i}$ is superfluous. In fact, by passing to normalizations, even the condition of normality of the $X_{i}$ can be omitted for the construction (cf. [096]). We are, however, interested in having an $\mathbb{N}_{0}^{n}$-valued valuation-like function and not merely a $\mathbb{Z}^{n}$-valued one. Note in particular, that if $v(s)=\left(a_{1}, \ldots, a_{n}\right)$, then, by the construction, the line bundle $\mathscr{L}^{k} \otimes \mathscr{L}_{1}^{-a_{1}} \otimes \cdots \otimes \mathscr{L}_{i}^{-a_{n-i+1}}$ over $X_{n-i+1}$ is effective for each $i \in\{1, \ldots, n\}$. This property will be crucial in the proof of the main result.

\section{A POLYhedRAl OKOUNKOV BODY}

Let $X$ be a projective normal $n$-dimensional complex variety and let $\mathscr{L} \rightarrow$ $X$ be an ample line bundle. Since Okounkov bodies satisfy the property $\Delta\left(\mathscr{L}^{k}\right)=k \Delta(\mathscr{L}), k \in \mathbb{N}$ (cf. [LM09, Proposition 4.1.]), we may without loss of generality assume that $\mathscr{L}$ is very ample.

By a Bertini-type theorem by Seidenberg (cf. [S50]), for a generic tuple $\left(\xi_{1}, \ldots, \xi_{n-1}\right)$ of sections $\xi_{1}, \ldots, \xi_{n-1} \in H^{0}(X, \mathscr{L})$ the zero sets

$$
X_{i}:=\left\{x \in X \mid \xi_{1}(x)=\cdots=\xi_{n-i}(x)=0\right\}, \quad i=1, \ldots, n-1
$$

define irreducible normal subvarieties of $X$ with $\operatorname{dim} X_{i}=i, i=1, \ldots, n-1$. Now, fix such a tuple $\left(\xi_{1}, \ldots, \xi_{n-1}\right)$ and let $X_{0}:=\{p\} \subseteq X_{1}$ for some point $p \in X_{1}$. Let

$$
v: \bigsqcup_{k \geq 0} H^{0}\left(X, \mathscr{L}^{k}\right) \backslash\{0\} \rightarrow \mathbb{N}^{n}
$$

be the valuation-like function defined by the flag

$$
X_{0} \subseteq X_{1} \subseteq \cdots \subseteq X_{n-1} \subseteq X_{n}:=X,
$$

and let $\Delta \subseteq \mathbb{R}^{n}$ be the associated Okounkov body. Similarly, let

$$
v_{1}: \bigsqcup_{k \geq 0} H^{0}\left(X_{1},\left.\mathscr{L}^{k}\right|_{X_{1}}\right) \backslash\{0\} \rightarrow \mathbb{N}
$$


be the valuation-like function defined by the flag $X_{0} \subseteq X_{1}$, and let $\Delta_{1} \subseteq \mathbb{R}$ be the corresponding Okounkov body. Then

$$
\Delta_{1}=[0, b] \subseteq \mathbb{R},
$$

where $b=\left.\operatorname{deg} \mathscr{L}\right|_{X_{1}}$ (cf. [LM09, Ex. 1.14]).

Lemma 3.1. The inclusion

$$
[0, b] \times\{0\} \times \cdots \times\{0\} \subseteq \Delta
$$

holds.

Proof. By the closedness of $\Delta$ it suffices to prove that $(c, 0, \ldots, 0) \in \Delta$ for every $0<c<b$.

By the ampleness of $\mathscr{L}$ there exists an $N_{0} \in \mathbb{N}$ such that the restriction maps

$$
R_{j}: H^{0}\left(X, \mathscr{L}^{j}\right) \rightarrow H^{0}\left(X_{1},\left.\mathscr{L}^{j}\right|_{X_{1}}\right)
$$

are surjective for $j \geq N_{0}$. If now $c \in(0, b)$, choose a point $v_{1}(\tau) / m \in(c, b)$ for a section $\tau \in H^{0}\left(X_{1},\left.\mathscr{L}^{m}\right|_{X_{1}}\right)$. Hence, for sufficiently large $N \in \mathbb{N}$, there exists a section $\widetilde{\tau_{N}} \in H^{0}\left(X, \mathscr{L}^{N m}\right)$ with $R_{N m}\left(\widetilde{\tau_{N}}\right)=\tau^{N}$. Then $v\left(\widetilde{\tau_{N}}\right)=$ $\left(v_{1}\left(\tau^{N}\right), 0, \ldots, 0\right)=\left(N v_{1}(\tau), 0, \ldots, 0\right)$, so that $\left(v_{1}(\tau) / m, 0, \ldots, 0\right) \in \Delta$. By the convexity of $\Delta$ we then have $(c, 0, \ldots, 0) \in \Delta$.

Remark 3.2. The lemma would of course also hold if the subvarieties $X_{i}$ were defined by unequal line bundles $\mathscr{L}_{i}, i=1, \ldots, n-1$. For a related result for line bundles $\mathscr{L}$ that are not ample, but merely big, cf. [J10, Theorem $\mathrm{B}]$.

Let $e_{1}, \ldots, e_{n}$ be the standard basis for $\mathbb{R}^{n}$.

Theorem 3.3. The Okounkov body $\Delta$ is the convex hull of the set

$$
\left\{0, b e_{1}, e_{2}, \ldots, e_{n}\right\} .
$$

Proof. First of all, $v\left(\xi_{n-i+1}\right)=e_{i}$ for $i=2, \ldots, n$. Since the linear system defined by $\mathscr{L}$ is basepoint-free, $0 \in v\left(H^{0}(X, \mathscr{L}) \backslash\{0\}\right)$. By Lemma 3.1 $b e_{1} \in \Delta$. Hence the convex hull of the points $0, b e_{1}, e_{2}, \ldots, e_{n}$ is a subset of $\Delta$.

It thus suffices to prove that for every $a=v(s)$ for some $s \in H^{0}\left(X, \mathscr{L}^{k}\right) \backslash$ $\{0\}$ there exist $x_{0}, \ldots, x_{n} \geq 0$ such that

$$
\begin{aligned}
& x_{0}+\cdots+x_{n}=k, \\
& a=x_{0} \cdot 0+x_{1} b e_{1}+x_{2} e_{2}+\cdots+x_{n} e_{n} .
\end{aligned}
$$

For this, let $a=\left(a_{1}, \ldots, a_{m}, 0, \ldots, 0\right)=v(s)$ for $s \in H^{0}\left(X, \mathscr{L}^{k}\right)$ and assume that $a_{m} \geq 1$. Then $\left.\left(s / \xi_{n-m+1}^{a_{m}}\right)\right|_{X_{m-1}}$ defines a section in $H^{0}\left(X_{m-1},\left.\mathscr{L}^{k-a_{m}}\right|_{X_{m-1}}\right)$, and by iteration we can write

$$
a=\left(a_{1}, 0, \ldots, 0\right)+\sum_{i=2}^{m} a_{i} v\left(\xi_{n-i+1}\right),
$$

where $a_{1}=v_{1}(t)$ for the section

$$
t=\left.\left(s /\left(\xi_{n-1}^{a_{2}} \cdots \xi_{n-m+1}^{a_{m}}\right)\right)\right|_{X_{1}} \in H^{0}\left(X_{1},\left.\mathscr{L}^{k-\sum_{i=2}^{m} a_{i}}\right|_{X_{1}}\right) .
$$


Since the line bundle $\left.\mathscr{L}^{k-\sum_{i=2}^{m} a_{i}}\right|_{X_{1}}$ is effective we must have $p:=k-$ $\sum_{i=2}^{m} a_{i} \geq 0$. Indeed, $\left.\mathscr{L}^{r}\right|_{X_{1}}$ is effective for every $r \geq 0$, and hence $\left.\mathscr{L}^{r}\right|_{X_{1}}$ cannot be effective for any $r<0$. Since $\Delta_{1}=[0, b]$ there exist real $x_{0}, x_{1} \geq 0$ with $x_{0}+x_{1}=p$ and

$$
a_{1}=x_{0} \cdot 0+x_{1} b
$$

and hence

$$
a_{1} e_{1}=x_{0} \cdot 0+x_{1} b e_{1} .
$$

By putting

$$
x_{i}:=a_{i} \quad i=2, \ldots, n,
$$

we have thus found nonnegative real numbers $x_{0}, \ldots, x_{n}$ satisfying (51).

\section{Global Okounkov bodies for homogeneous surfaces}

In this section we prove that, if $X$ is a homogeneous projective surface admitting a rationally polyhedral pseudoeffective cone, the global Okounkov body, with respect to a suitably chosen flag of subvarieties, is a rational polyhedral cone.

Let $X$ be a normal projective complex variety. Let $N^{1}(X)_{\mathbb{R}} \cong \mathbb{R}^{r}$, where $r$ is the Picard number of $X$, be its real Néron-Severi space, and let $\overline{\operatorname{Eff}}(X) \subseteq$ $N^{1}(X)_{\mathbb{R}}$ be the pseudoeffective cone of $X$.

Let $X_{0} \subseteq \cdots \subseteq X_{n}=X$ be a flag of irreducible subvarieties as in the previous sections. We recall that the global Okounkov body, $\Delta_{X \bullet}(X)$, of $X$ with respect to the above flag is the closed convex cone in $\mathbb{R}^{n} \oplus N^{1}(X)_{\mathbb{R}}$ generated by the semigroup

$$
\begin{aligned}
S(X, v) & :=\left\{(v(s),[L]) \mid L \quad \text { big line bundle, } s \in H^{0}(X, L) \backslash\{0\}\right\} \\
& \subseteq \mathbb{N}_{0}^{n} \oplus N^{1}(X)_{\mathbb{Z} .}
\end{aligned}
$$

(cf. [LM09]). Here there valuation-like function is extended to the (nonzero homogeneous elements of) ring generated by the spaces of sections of all effective line bundles on $X$.

In order to adapt the idea of the proof of the main theorem in the previous section to a global situtation, we first need the following lemma.

Lemma 4.1. Let $X$ be a complex normal projective variety, and let $X \bullet$ be an admissible flag of subvarieties. If $L$ is a nef line bundle on $X$, the inclusion

$$
\left[0,\left(L \cdot X_{1}\right)\right] \times\{0\}^{n-1} \subseteq \Delta_{X \bullet}(L)
$$

holds.

Proof. First of all, we note that, by Serre vanishing, for any ample line bundle $E$, the restriction map

$$
R^{k}: H^{0}\left(X, E^{k}\right) \rightarrow H^{0}\left(X_{1},\left.E^{k}\right|_{X_{1}}\right)
$$

is surjective for sufficienly big $k \in \mathbb{N}$.

To simplify the notation, we will now work with the corresponding Weil divisor. Hence, let $F$ be a Weil divisor, such that $\mathcal{O}_{X}(F)=L$. 
It now suffices to prove that $(a / j, 0, \ldots, 0) \in \Delta_{X \bullet}(F)$, for

$$
a \in v_{1}\left(H^{0}\left(X_{1},\left.\mathcal{O}_{X}(j F)\right|_{X_{1}}\right)\right), \quad j \in \mathbb{N}_{0},
$$

where $v_{1}$ is the valuation-like function, defined on (the nonzero homogenous elements of) the ring of all sections of all effective line bundles on $X_{1}$, with respect to the point $X_{0} \in X_{1}$. For such an $a$, we have

$$
m a \in v_{1}\left(H^{0}\left(X_{1},\left.\mathcal{O}_{X}(m j F+D)\right|_{X_{1}}\right)\right), \quad m \in \mathbb{N},
$$

since $0 \in v_{1}\left(H^{0}\left(X_{1},\left.\mathcal{O}_{X}(D)\right|_{X_{1}}\right)\right)$ because $D$ is ample. Since $m j F+D$ is ample, $(m a, 0, \ldots, 0) \in \Delta_{X} .(m j F+D)$ by Lemma 3.1. Hence,

$$
(a, 0, \ldots, 0) \in \Delta_{X}\left(j F+\frac{D}{m}\right), \quad m \in \mathbb{N},
$$

In particular, $\left((a, 0), j F+\frac{D}{m}\right) \in \Delta_{X}(X)$, for every $m \in \mathbb{N}$. Since $\Delta_{X \bullet}(X)$ is closed, we thus have $\lim _{m \rightarrow \infty}\left((a, 0), j F+\frac{D}{m}\right)=((a, 0), j F) \in \Delta_{X}$. $(X)$ (cf. also [AKL12, Lemma 8]). Hence, $(a, 0, \ldots, 0) \in \Delta_{X}(j F)$, so that

$$
(a / j, 0, \ldots, 0) \in \Delta_{X \bullet}(F) .
$$

Remark 4.2. If $L$ is basepoint-free, the conclusion of Lemma 4.1, for a flag $X_{\bullet}$ defined by a very general choice of ample divisors $\left(D_{1}, \ldots, D_{n}\right)$, also follows from [J10, Theorem B]. Indeed, if $L$ is basepoint-free, the restricted volume on the left hand side of [J10, Theorem B] is given by the intersection number $\left(L \cdot X_{1}\right)$ (cf. [J10, Cor. 3.3.]).

We are now ready to state the main result of this section. Recall that a variety $X$ is called homogeneous if $X$ carries a transitive action of a connected algebraic group $G$.

Theorem 4.3. Let $X$ be a homogenous projective surface with a rational polyhedral pseudoeffective cone $\overline{E f f}(X)$, and let $\xi_{1}, \ldots, \xi_{r}$ be integral generators of $\overline{E f f}(X)$. Let $D$ be a very ample divisor on $X$. For a generic choice of flag $X_{0} \subseteq X_{1}$, where $X_{1} \in|D|$, the global Okounkov body $\Delta_{X \bullet}(X)$ is the rational polyhedral cone generated by the vectors

$$
\left((0,0), \xi_{1}\right),\left(\left(d\left(\xi_{1}\right), 0\right), \xi_{1}\right), \ldots,\left((0,0), \xi_{r}\right),\left(\left(d\left(\xi_{r}\right), 0\right), \xi_{r}\right),((0,1),[D]),
$$

where $d\left(\xi_{j}\right)$ is the degree of $\xi_{j}$ along the curve $X_{1}$.

Proof. First of all, every effective line bunde on $X$ is nef (cf. [L04, Example 1.4.7.]), and, hence, Lemma 4.1 holds for every effective line bundle on $X$.

Moreover, there is a countable family $\left\{L_{j}\right\}_{j \in \mathbb{N}}$ of line bundles on $X$ such that each line bundle $L$ is numerically equivalent to some $L_{j}$. In particular, for any flag $X_{\bullet}$ of subvarieties, the global Okounkov body $\Delta_{X \bullet}(X)$ can be defined using only the line bundles $L_{j}, j \in \mathbb{N}$. We can without loss of generality assume that the $L_{j}$ form a lattice, and that this lattice contains all divisors $k D$, for $k \in D$. Now, for a generic tuple $\left(X_{0}, D_{1}, \ldots, D_{n-1}\right) \in$ $X \times|D|^{n-1}$, with $X_{0} \in D_{1} \cap \cdots \cap D_{n-1}$, Lemma 4.1 holds for each effective $L_{j}$. Under these assumptions we now have

$$
\left[0, d\left(\xi_{j}\right)\right] \times\{0\} \subseteq \Delta_{X \bullet}\left(\xi_{j}\right) \subseteq \Delta_{X}(X), \quad j=1, \ldots, r .
$$


Hence, the global Okounkov body $\Delta_{X \bullet}(X)$ contains all the vectors in the claim.

In order to prove that the given vectors generate the cone $\Delta_{X} .(X)$, let $(v(s),[L]) \in S(X, v)$, with $v(s)=\left(a_{1}, a_{2}\right)$. Then, $E:=L \otimes \mathcal{O}_{X}\left(-a_{2} D\right)$ is effective. Hence,

$$
[E]=t_{1} \xi_{1}+\cdots+t_{r} \xi_{r}
$$

for some $t_{1}, \ldots, t_{r} \geq 0$. The Okounkov body, with respect to the point $X_{0}$, of the restriction of $E$ to $X_{1}$ is given by

$$
\Delta_{X_{0}}\left(\left.E\right|_{X_{1}}\right)=\left[0,\left(E \cdot X_{1}\right)\right] .
$$

Hence, $a_{1} \in\left[0,\left(E \cdot X_{1}\right)\right]$, so that $a_{1}=c\left(E \cdot X_{1}\right)$, for some $c \in[0,1]$. Since

$$
\left(E \cdot X_{1}\right)=t_{1} d(\xi)+\cdots+t_{r} d\left(\xi_{r}\right)
$$

we have

$$
a_{1}=\sum_{i=1}^{r} c t_{i} d\left(\xi_{i}\right)
$$

It follows that

$\left(\left(a_{1}, a_{2}\right),[E]\right)=\sum_{i=1}^{r} t_{i}(1-c)\left((0,0), \xi_{i}\right)+\sum_{i=1}^{r} c t_{i}\left(\left(d\left(\xi_{i}\right), 0\right), \xi_{i}\right)+a_{2}((0,1),[D])$.

This shows that the closed convex cone generated by the vectors in the claim contains the semigroup $S(X, v)$, and this proves the theorem.

Example 1. If $X=\mathbb{P}^{1} \times \mathbb{P}^{1}$ with projections $p_{1}, p_{2}$ onto the respective factors, the pseudoeffective cone is generated by the divisors $D_{1}$ and $D_{2}$, where $\mathcal{O}_{X}\left(D_{i}\right)=p_{i}^{*} \mathcal{O}(1), i=1,2$. The intersection relations are given by

$$
D_{1}^{2}=D_{2}^{2}=0, \quad D_{1} \cdot D_{2}=1 \text {. }
$$

If we choose the ample divisor as $D:=D_{1}+D_{2}$, the global Okounkov body with respect to a generic flag is thus given as the closed cone generated by the vectors

$$
((0,1),[D]),\left((0,0),\left[D_{1}\right]\right),\left((0,0),\left[D_{2}\right]\right),\left((1,0),\left[D_{1}\right]\right),\left((1,0),\left[D_{2}\right]\right) .
$$

Example 2. If $D$ is the ample divisor of Theorem 4.3, we know from Theorem 3.3 that the Okounkov body $\Delta_{X} \cdot\left(\mathcal{O}_{X}(D)\right)$ of $D$ is the triangle generated by the vectors $(0,0),(D \cdot D, 0)$, and $(0,1)$. In order to see that $\Delta_{X \bullet}\left(\mathcal{O}_{X}(D)\right)=\Delta_{X}(D)$ occurs as a slice of the global Okounkov body $\Delta_{X} .(X)$, it suffices to see that the vector $((D \cdot D, 0),[D])$ is a nonnegative linear combination of the generators in Theorem 4.3. For this, write $[D]$ as $[D]=t_{1} \xi_{1}+\cdots+t_{r} \xi_{r}$, with $t_{1}, \ldots, t_{r} \geq 0$. Then $D \cdot D=$ $\sum_{i=1}^{r} t_{i} \xi_{i} \cdot D=\sum_{i=1}^{r} t_{i} d\left(\xi_{i}\right)$, so that $((D \cdot D, 0),[D])=\sum_{i=1}^{r} t_{i}\left(\left(d\left(\xi_{i}\right), 0\right), \xi_{i}\right)$. This shows that $\Delta_{X \bullet}(D)$ is the preimage of $[D]$ under the map $\Delta_{X \bullet}(X) \rightarrow$ $\overline{\mathrm{Eff}}(X), \quad(x, y) \mapsto y$.

Remark 4.4. The only surface which occurs as a flag variety $G / P$, for a complex reductive group $G$ and a parabolic subgroup $P$, is $\mathbb{P}^{1} \times \mathbb{P}^{1}$. On the other hand, Bauer gives a classification of the abelian surfaces admitting a rational polyhedral pseudoeffective cone in [B97]. 


\section{Multiplicities For Group RePresentations}

The purpose of this section is to apply the result from the previous sections to a setting occurring in the context of representations of complex reductive groups.

Let $X$ be a normal projective variety, and let $\mathscr{L} \rightarrow X$ be an ample line bundle. Assume that $L$ is a complex reductive group which acts on $X$ and that this action lifts to an action on $\mathscr{L}$ by bundle automorphisms. Then there is a natural representation of $L$ on each space $H^{0}\left(X, \mathscr{L}^{k}\right)$, for $k \in \mathbb{N}_{0}$. We are then interested in finding the decomposing under $L$ of $H^{0}\left(X, \mathscr{L}^{k}\right)$;

$$
H^{0}\left(X, \mathscr{L}^{k}\right)=\bigoplus_{\mu} m(k, \mu) W_{\mu} .
$$

Here $W_{\mu}$ is the irreducible $L$-representation of highest weight $\mu$, and $m(k, \mu)=$ $\operatorname{dim}_{\mathbb{C}} \operatorname{Hom}_{L}\left(W_{\mu}, H^{0}\left(X, \mathscr{L}^{k}\right)\right)$ its multiplicity in $H^{0}\left(X, \mathscr{L}^{k}\right)$.

To find the decomposition above is in general a very hard problem and only a few special cases are known. Instead, it has turned out to be fruitful to consider decompositions asymptotically in a certain sense. Indeed, if $m(1, \mu) \neq 0$, then $m(k, k \mu) \neq 0$ for all $k \in \mathbb{N}$. Moreover, the multiplicity $m(k, k \mu)$ is a polynomial function of $k$ (cf. [096]), where the leading coefficient is given by the Euclidean volume of a certain compact convex set, namely of a slice of an Okounkov body for a line bundle defined by intersecting the Okounkov body with a certain affine subspace. However, this body need not in general be polyhedral. In the sequel we will see that the leading coefficient of $m(k, k \mu)$ can be interpreted as the volume of a polyhedral Okounkov body for an ample line bundle, depending on $\mu$, and, hence, also as the self-intersection number of this line bundle.

By the Borel-Weil theorem, the highest weight representation $W_{\mu}$ can be realized geometrically as the space of sections of a line bundle as

$$
W_{\mu} \simeq H^{0}\left(Y_{\mu}, \mathscr{L}_{\mu}\right)
$$

where $Y_{\mu}=L / Q_{\mu}$ is a flag variety for $L$ and $\mathscr{L}_{\mu} \rightarrow Y_{\mu}$ is an ample $L$ equivariant line bundle. The multiplicities $m(k, k \mu)$ can now also be given a geometric interpretation. For this, let $Y_{\mu}$ denote the variety having the same point-set as $Y_{\mu}$, but equipped with the opposite complex structure, i.e., the structure sheaf of $Y_{\mu}^{-}$is given by the complex-conjugate of the structure sheaf of $Y_{\mu}$. The variety $Y_{\mu}$ can also be described as the flag variety $Y_{\mu}^{-}=L / Q_{\mu}^{-}$, where $Q_{\mu}^{-}$is the opposite parabolic subgroup of $Q_{\mu}$. Moreover, let $T \subseteq Q_{\mu} \cap Q_{\mu}^{-}$be a maximal torus such that the line bundle $\mathscr{L}_{\mu}$ is defined as

$$
\mathscr{L}_{\mu}=L \times_{\chi(\mu)} \mathbb{C}
$$

for a character $\chi(\mu): T \rightarrow \mathbb{C}^{\times}$extended uniquely to a character of $Q_{\mu}$ (which we also denote by $\chi(\mu)$ ). Now we can defined the line bundle

$$
\mathscr{L}_{\mu}^{-}:=L \times_{\chi^{-}(\mu)} \mathbb{C}
$$

by extending the character $\chi(\mu): T \rightarrow \mathbb{C}^{\times}$to a character $\chi(\mu): Q_{\mu}^{-} \rightarrow \mathbb{C}^{\times}$ of $Q_{\mu}^{-}$. The multiplicity $m(k, k \mu)$ is then given by

$$
m(k, k \mu)=\operatorname{dim} H^{0}\left(X \times Y_{\mu}^{-}, \mathscr{L}^{k} \otimes\left(\left(\mathscr{L}_{\mu}^{-}\right)^{k}\right)^{*}\right)^{L},
$$


where $H^{0}\left(X \times Y_{\mu}^{-}, \mathscr{L}^{k} \otimes\left(\left(\mathscr{L}_{\mu}^{-}\right)^{k}\right)^{*}\right)^{L}$ denotes the space of $L$-invariant sections of the exterior tensor product bundle $\mathscr{L}^{k} \otimes\left(\left(\mathscr{L}_{\mu}^{-}\right)^{k}\right)^{*}$ over $X \times Y_{\mu}^{-}$.

With the aim of putting the multiplicity formula above into the framework of spaces of global sections, the multiplicity can also be described as the dimension of the space of all sections of a coherent sheaf;

$$
m(k, k \mu)=\operatorname{dim} H^{0}(Z(\mu), \mathcal{L}(k, \mu)) .
$$

Here $Z(\mu):=X \times Y_{\mu}^{-} / / L$ is the GIT-quotient of $X \times Y_{\mu}^{-}$with respect to the line bundle $\mathscr{L} \otimes\left(\mathscr{L}_{\mu}^{-}\right)^{*}$ and the action of $L$. For a thorough treatment of this result (in the case when $X$ is nonsingular), we refer to [S95], where the space $Z(\mu)$ is also proven to be homeomorphic to the symplectic quotient coming from the natural moment map

$$
\mu: X \times Y_{\mu}^{-} \rightarrow \mathfrak{k}^{*},
$$

where $\mathfrak{k}$ is the Lie algebra of a maximal compact subgroup $K$ of $L$ which acts on $X \times Y_{\mu}^{-}$in a Hamiltonian fashion. The sheaf $\mathcal{L}(k, k \mu)$ is naturally given as the $L$-invariants of the direct image sheaf $\pi_{*}\left(\mathscr{L}^{k} \otimes\left(\left(\mathscr{L}_{\mu}^{-}\right)^{k}\right)^{*}\right)$. Here $\pi$ is the quotient map onto $Z(\mu)$ from the set of semi-stable points of $X \times Y_{\mu}^{-}$. The sheaf $\mathcal{L}(k, k \mu)$ is a coherent sheaf, although not in general a line bundle. However, there exists a natural number $q$, such that for all $k \in \mathbb{N}$, the $k q$-th tensor power $\left(\mathscr{L} \otimes\left(\mathscr{L}_{\mu}^{-}\right)^{*}\right)^{k q}$ descends to a line bundle $\mathcal{L}(k q, k q \mu)$ by the above construcion of taking $L$-invariants of the direct image sheaf under $\pi$. Moreover, $q$ can even be chosen so that the line bundle $\mathcal{L}(q, q \mu)$ is ample (cf. [S95, Thm 2.17]).

Even though $X \times Y_{\mu}^{-}$is a nonsingular variety when $X$ is nonsingular, the quotient $Z(\mu)$ is in general singular. However, it has rational singularities, so it is in particular a normal variety (cf. [S95]).

We can now apply the result from the previous section to this setting where $X=Z(\mu)$ and $\mathscr{L}=\mathcal{L}(q, q \mu)$. We then have the following result.

Theorem 5.1. Let

$$
Z_{0} \subseteq Z_{1} \subseteq \cdots \subseteq Z_{n}=Z(\lambda, \mu),
$$

where $n=\operatorname{dim} Z(\mu)$ be a flag of irreducible normal subvarieties defined by a generic tuple $\left(\xi, \ldots, \xi_{n-1}\right)$ of sections $\xi_{1}, \ldots, \xi_{n} \in H^{0}(Z(\mu), \mathcal{L}(q, q \mu))$ and a point $Z_{0} \in Z_{1}$ and let $\Delta=\operatorname{conv}\left\{0, b e_{1}, e_{2}, \ldots, e_{n}\right\}$ be the associated Okounkov body for the line bundle $\mathcal{L}(q, q \mu)$. Then

$$
\lim _{k \rightarrow \infty} \frac{m(k q, k q \mu)}{k^{n}}=\operatorname{Vol}(\Delta)=b .
$$

Moreover, the volume $\operatorname{Vol}(\Delta)=b$ is also given by the self-intersection number of the ample divisor $\mathcal{L}(q, q \mu)$.

A particularly interesting special case of the above setting is the one when $X=G / P$ is a flag variety of a complex reductive group $G$ containing $L$ as a subgroup, and $\mathscr{L}=\mathscr{L}_{\lambda}$ is a homogeneous line bundle, so that the space of sections $H^{0}\left(X, \mathscr{L}_{\lambda}^{k}\right)$ is the Borel-Weil realization of the irreducible $G$-module $V_{k \lambda}$ of highest weight $k \lambda$. The decomposition under $L$ then amounts to the 
branching law

$$
V_{k \lambda}=\sum_{\mu} m(k \lambda, \mu) W_{\mu}
$$

Remark 5.2. The part of the main theorem which describes the asymptotics of the multiplicity function $m(k q, k \mu)$ as a self-intersection number can also be derived from the Riemann-Roch theorem for singular varieties, given vanishing results for the higher cohomology groups of the sheaf $\mathcal{L}(q, q \mu)$. Moreover, irrespective of the shape of the Okounkov body on the GITquotient, the asymptotic multiplicity would be given as the self intersection number of a divisor. However, the polyhedral nature of the Okounkov body $\Delta$ above also allows us, for fixed $k \in \mathbb{N}$, to approximate the multiplicity $m(k q, k q \mu) 1$ by counting the number of integral points of the convex polytope $k \Delta$. For a precise formulation of the meaning of this approximation, we refer to [KK09, Cor. 1.5], which is concerned with comparing the points of a semigroup with that of its saturation with respect to the closed convex cone generated by the semigroup. In this sense, our result fits into a general philosophy of seeking polyhedral expressions for multiplicities (cf. [L98], [BZ01]).

\section{Concluding REMarks}

The subvarieties $X_{i}$ occurring in the flag (44) are unfortunately only defined very implicitly. It would be interesting to find more explicit examples of admissible flags yielding polyhedral Okounkov bodies. In particular, in the original setting developed by Okounkov, involving the presence of a group action, it is desirable to have a flag which is invariant under a Borel subgroup in order to relate the Okounkov body to asymptotics of multiplicities for irreducible representations. In particular, this is crucial for being able to work with a fixed Okounkov body, for which certain slices describe multiplicities for subrepresentations, as opposed to using a variety and a line bundle which depend on $\mu$ for constructing a $\mu$-dependent Okounkov body in order to treat the multiplicities $m(k, k \mu)$. However, the condition of having an invariant flag is not well compatible with our approach using a flag where each $X_{k}$ is a complete intersection of divisors. Indeed, one can not hope for a Bertini-type theorem which provides invariant flags. For instance, in the case of a flag variety $X=G / P$, the flags of Borel-invariant subvarieties of $X$ consist of Schubert varieties, and an inclusion $X_{k} \subseteq X_{k+1}$ of Schubert varieties is not in general given by a Cartier divisor.

\section{REFERENCES}

[AKL12] Anderson, D., Küronya, A., Lozovanu, V., Okounkov bodies of finitely generated divisors, International Mathematics Research Notices 2013; doi: 10.1093/imrn/rns286

[B97] Bauer, T., Seshadri constants of quartic surfaces, Math. Ann. 309 (1997), 3, 475-481

[BZ01] Berenstein, A., Zelevinsky, A., Tensor product multiplicities, canonical bases and totally positive varieties, Invent. Math. 143 (2001), 1, 77-128

[B86] Brion, M., Sur l'image de l'application moment, Séminaire d'algèbre Paul Dubreil et Marie-Paule Malliavin (Paris, 1986), Lecture Notes in Math., 1296, 177-192, Springer, Berlin, 1987 
[J10] Jow, Shin-Yao, Okounkov bodies and restricted volumes along very general curves, Adv. Math. 223, (2010), 4, 1356-1371

[K11] Kaveh, K., Crystal bases and Newton-Okounkov bodies, preprint, arxiv.org/abs/1101.1687

[KK09] Kaveh, K., Khovanskii, A.G., Newton convex bodies, semigroups of integral points, graded algebras and intersection theory, preprint, arxiv.org/abs/0904.3350

[KK10] Kaveh, K., Khovanskii, A.G., Convex bodies associated to actions of reductive groups, preprint, arxiv.org/abs/1001.4830

[L04] Lazarsfeld, R., "Positivity in Algebraic Geometry I", Springer, 2004

[LM09] Lazarsfeld, R., Mustaţă, M., Convex bodies associated to linear series, Ann. Sci. Éc. Norm. Supér. 42 (2009), 783-835

[L98] Littelmann, P., Cones, crystals, and patterns, Transform. Groups 3 (1998), 2, $145-179$

[O96] Okounkov, A., Brunn-Minkowski inequality for multiplicities, Invent. Math. 125 (1996), 405-411

[S50] Seidenberg, A., The hyperplane sections of normal varieties, Trans. Amer. Math. Soc. 69 (1950), 357-386

[SS14] Schmitz, D., Seppänen, H., On the polyhedrality of global Okounkov bodies, in preparation

[S95] Sjamaar, R., Holomorphic slices, symplectic reduction and multiplicities of representations, Ann. of Math. (2) 141 (1995), no. 1, 87-129

Henrik Seppänen, Mathematisches Institut, Georg-August-Universität Göttingen, Bunsenstrasse 3-5, D-37073 Göttingen, Germany

E-mail address: hseppaen@uni-math.gwdg.de 